\title{
Belgeo
}

Revue belge de géographie

3 | 2014

Art(s) \& Espace(s) / Art(s) \& Space(s)

\section{L'artiste, la carte et le territoire : détourner et retourner les représentations}

The artist, the map and the territory. Distorting visual representations

\section{Elsa Vivant, Burcu Ozdirlik et Nadia Arab}

\section{(2) OpenEdition}

Journals

Édition électronique

URL : http://journals.openedition.org/belgeo/13286

DOI : 10.4000/belgeo.13286

ISSN : 2294-9135

Éditeur :

National Committee of Geography of Belgium, Société Royale Belge de Géographie

Référence électronique

Elsa Vivant, Burcu Ozdirlik et Nadia Arab, «L'artiste, la carte et le territoire : détourner et retourner les représentations », Belgeo [En ligne], 3 | 2014, mis en ligne le 19 décembre 2014, consulté le 19 avril 2019. URL : http://journals.openedition.org/belgeo/13286; DOI : 10.4000/belgeo.13286

Ce document a été généré automatiquement le 19 avril 2019.

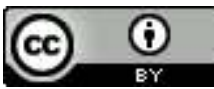

Belgeo est mis à disposition selon les termes de la licence Creative Commons Attribution 4.0 International. 


\title{
L'artiste, la carte et le territoire : détourner et retourner les représentations
}

\author{
The artist, the map and the territory. Distorting visual representations
}

\author{
Elsa Vivant, Burcu Ozdirlik et Nadia Arab
}

1 Le monde de la géographie accorde un intérêt croissant au travail des artistes pour repenser les modalités et méthodologies de production et de représentation des savoirs territoriaux (Blanc, 2008; Volvey, 2007 ; Grésillon, 2008). Les interventions d'artistes en lien avec les territoires et leurs enjeux sont nombreuses et variées: mise en forme de données géo-localisées (O'Rourke, 2013), intervention en contexte réel (Ardenne, 2002), animation de l'espace public (Chaudoir, 2000), mise en forme de récits des épreuves de la migration (Mekdjian, Amilhat Szary et al., 2014), canulars et détournement artisticopolitiques d'enjeux territoriaux (Gonon, 2011), et enfin, détournement des procédés cartographiques. Dans la continuité de ces travaux, cet article explore de nouvelles approches de l'intervention artistique en lien avec le territoire, où l'intervention de l'artiste ne vise plus (ou pas uniquement) à faire œuvre mais à contribuer aux réflexions sur les transformations territoriales en cours ou à venir. Dans les démarches étudiées ${ }^{1}$, les artistes sortent de leur rôle habituel de créateurs d'œuvres d'art pour contribuer, aux côtés des acteurs du territoire (professionnels de l'action territoriale, élus, habitants, usagers...), à la production de connaissances sur le territoire, à l'identification et à la représentation de problèmes et d'enjeux locaux, au dialogue et à la concertation avec la population locale, à l'élaboration des choix de transformation des espaces. Les interventions artistiques sont de différentes natures : la production d'un spectacle de rue sur la sensibilisation au risque inondation; l'expérimentation d'une œuvre-enquête pour comprendre les modes d'appropriation des espaces publics ; la création d'un lieu de rencontre et de sociabilité comme instrument d'éducation populaire et de développement social dans le cadre d'une opération ANRU ; la réalisation d'une base de données territorialisées et sa mise en forme sur des supports numériques pour valoriser 
le patrimoine. Dans les quatre cas étudiés, l'usage de la cartographie apparaît comme une des modalités d'intervention des artistes: mise à l'épreuve sur le terrain des cartes de planification, analyse d'un objet par une cartographie des liens le reliant à son environnement, formation des habitants à la lecture et à la production de cartes, mise en place d'une carte numérique interactive. En présentant chaque exemple dans son contexte d'intervention, il s'agit d'interroger les usages que font les artistes de la carte.

Ces expériences renvoient aux débats soulevés par le courant de la cartographie critique et les mouvements de cartographie participative ${ }^{2}$ (néocartographie, cartographie autochtone, etc.). Les tenants d'une cartographie critique rappellent que les cartes ne sont pas des relevés passifs d'informations territoriales mais qu'elles sont chargées de valeurs idéologiques et d'intentionnalité (la conquête ou le contrôle d'un territoire par exemple) et traduisent des rapports de force. Elles ont un impact sur la pensée des acteurs qui les utilisent (Debardieux, Lardon, 2004 ; Le Bourhis, 2007 ; Lascoumes, 2007 ; Hirt, Roche, 2013). Instrument du pouvoir, la cartographie a longtemps été une affaire de professionnels, ce à quoi s'opposent les militants de la cartographie participative. La mise en œuvre de nouveaux outils numériques de représentation, la valorisation des logiques contributives et collaboratives, les dispositifs de formation des usagers et la reconnaissance de leurs compétences, la montée en puissance de la représentation visuelle dans l'expression des enjeux territoriaux conduisent à l'émergence d'une nouvelle figure du cartographe amateur (Joliveau, Noucher et al., 2013). Comme dans d'autres sphères de production de savoir et d'échange d'informations, l'élévation du niveau de formation et les possibilités offertes par le web contributif promeuvent la démocratisation des compétences à travers la figure du professionnel-amateur qui développe ses activités amateurs selon des standards professionnels (Flichy, 2010). Pour autant, le déploiement de cartographies alternatives (amateurs ou participatives) se heurte aux logiques économiques des opérateurs de production, gestion et visualisation des données et par les habitudes, réflexes et intérêts des professionnels de la cartographie et de l'action territoriale. Les interventions des artistes seront étudiées ici au regard de ces controverses sur la cartographie comme construction sociale porteuse de conventions plus ou moins négociées. La carte étant un instrument conventionnel de représentation et de collaboration des professionnels de l'action territoriale, leurs réactions révèlent si et en quoi les propositions cartographiques de ces artistes renouvellent les controverses sur la cartographie.

\section{Les cartes de zonage à l'épreuve du terrain}

3 Dans cette ville moyenne du Val de Loire, l'inondation est un événement aussi rare que dévastateur. La dernière crue majeure date de plus d'un siècle et les mémoires humaines l'ont oubliée. Les professionnels du risque peinent à diffuser une culture du risque et à faire prendre conscience aux habitants de la réalité de la menace et de ses conséquences. Dans ce contexte, et alors que les acteurs locaux révisent le plan de prévention du risque inondation (PPRI), ArtUrbain, opérateur de production et de diffusion de projets artistiques dans l'espace public, propose aux acteurs concernés de faire intervenir des artistes autour de la question de la sensibilisation au risque inondation. Au cours de l'année de préparation du projet artistique, les artistes et ArtUrbain rencontrent différents professionnels concernés par le risque inondation (pompiers, assureurs, météorologues, planificateurs,...) et différentes institutions qui leur ont présenté leur rôle 
dans la gestion des inondations, les enjeux et problèmes spécifiques au contexte local, les règles et documents relatifs aux usages de l'espace et la gestion de la crise. Lors de leurs repérages, les artistes, qui ne connaissaient ni la ville ni les enjeux de l'inondation, confrontent leur perception de l'espace, du fleuve et des dangers qu'il représente (ou pas) aux explications, parfois divergentes, des acteurs. Grâce à ces multiples rencontres, les artistes construisent leur propre culture du risque inondation et nourrissent leur création.

4 Leur projet artistique, auquel participent une centaine de personnes, propose une expérience quasi documentaire et immersive d'une ville faisant face à une crue, et se décompose en dix-huit activités se déroulant sur 24 heures. Certaines activités ont comme objet de montrer la technicité de la question et la diversité des approches du risque. Ainsi, après avoir présenté, lors de conférences volontairement jargonneuses animées par des professionnels concernés, les enjeux d'un PPRI, les modalités de sa réalisation et les intérêts en présence, le document de planification est mis à l'épreuve du terrain. En effet, au cours d'une promenade en ville, carte du PPRI en main, les artistes avaient constaté certaines incohérences dans la cartographie des zones à risques.

«On avait envie, à un moment, qu'il y ait une action qui concrétise ce rapport entre la carte et le terrain, qui questionne ces trucs de zonage, qui rende concret en fait, parce que la carte du coup cela devient aussi une réalité, le PPRI, ce n'est pas seulement un bout de papier, c'est aussi des zones de la ville qui sont concernées différemment par le risque inondation, qui ne vont pas avoir les mêmes dispositifs d'évacuation, les mêmes règles de construction, etc. » (artiste)

5 Les artistes ont conçu un dispositif qui met en scène les imperfections du zonage. Les participants marchent, en file indienne, le long d'une ligne tracée au sol à la craie. Cette ligne reprend le tracé des limites d'une zone inondable du PPRI, qui passe au milieu d'une rue plane dont un côté est classé en zone inondable, et l'autre pas. Les participants marchent ainsi de part et d'autre de la limite d'une zone à risque, chaussés côté inondable d'une botte, côté non-inondable d'une sandale de plage. Cette balade met à l'épreuve du contexte réel les délimitations technico-administratives de débordement du fleuve. Elle confronte la limite abstraite de la zone inondable (tracée sur une carte à partir de données historiques), et l'espace concret (une rue plate dont visuellement on ne voit pas pourquoi un côté serait inondable et l'autre pas). Marcher sur les limites du PPRI est ainsi un moyen de révéler le hiatus entre carte et réalité matérielle et de réinterroger les modes de production technico-administratives des documents de planification. En effet, l'usage de la cartographie comme instrument de gouvernement des risques laisse de larges zones d'interprétation que révèlent les incohérences des cartes. Celles-ci sont produites sur la base de savoirs flous (la mémoire, les traces) et des savoirs techniques marqués par l'incertitude (extrapolation des niveaux et effets des crues). Les cartes des risques résultent ensuite d'une négociation entre différents acteurs, où sont pris en considération différents enjeux politiques et économiques (valeurs foncières, possibilités d'urbanisation, etc.) (Le Bourhis, 2007). Les imprécisions de ces cartes, dont les effets réglementaires sont, eux, précis et contraignants, brouillent le sens du risque qu'elles sont censées représenter. Dans le cas étudié, l'intervention des artistes participe aux controverses sur les modes de productions des cartes en questionnant leur rapport à la réalité. En projetant dans l'espace la traduction effective de la cartographie du risque inondation, les artistes interpellent les professionnels dans leurs propres systèmes de représentations et interrogent le sens même des zonages opérés. A l'image d'autres 
artistes (Amilhat Szary, 2012), cette proposition artistique interroge la construction des limites et ce qu'elles séparent.

\section{Cartographier les pratiques d'un espace pour rendre compte de sa complexité}

6 La ville de Rosière, en proche banlieue parisienne, est l'objet d'une spectaculaire opération de renouvellement urbain depuis une dizaine d'années. Une attention particulière a été portée à la requalification des espaces publics, notamment par la réalisation de nouveaux espaces verts. Pourtant, ceux-ci semblent délaissés par les habitants qui les fréquentent peu. Pour comprendre les modes d'appropriation des espaces publics, les services techniques de la ville sollicitent la formation ArtPol dont le projet pédagogique est fondé sur la rencontre entre artistes et chercheurs en sciences sociales pour travailler sur des problèmes concrets de représentation et de formulation d'un problème. ArtPol s'adresse à des professionnels (principalement artistes et chercheurs) qui souhaitent prendre de la distance par rapport à leurs pratiques habituelles. Les réflexions théoriques sont mises en application à travers les projetsétudiants (travail en groupe pluridisciplinaire en réponse à une demande d'un commanditaire) où l'intervention des artistes-étudiants ne consiste pas en la création d'une œuvre d'art mais plutôt d'une " œuvre-enquête ». L'art est ici considéré comme une manière de répondre à des problèmes publics.

7 Quatre étudiantes ont choisi d'intervenir sur le problème présenté par la ville de Rosière, qui sera progressivement reformulé en « étude des attachements d'un lieu à venir ». Elles ont mis en place plusieurs dispositifs d'enquête et de restitution, inaboutis pour la plupart, qui relèvent soit de l'adaptation de leurs procédés artistiques habituels, soit de réponse à des exercices demandés dans le cadre de la formation. Elles mettent ainsi en œuvre un CartoAttaches, démarche qui s'inspire de la théorie de l'acteur-réseau (Latour, 2006). Elles choisissent de cartographier tout (objet ou individu) ce qui est relié, d'une manière ou d'une autre, au monument aux morts déplacé à plusieurs reprises pendant l'opération de rénovation urbaine. Pour cela, les artistes cherchent à identifier les personnes concernées par le monument aux morts ou impliquées dans son déplacement, pour dresser la liste des liens et des pratiques qui s'organisent autour lui. C'est ce que représente le CartoAttaches.

8 Un CartoAttaches n'a pas de forme ni de montage prédéfinis (c'est-à-dire une manière d'organiser et de représenter l'information pour montrer les liens). Ici, autour d'un croquis de la statue, gravitent dans un nuage de mots des noms d'objets ("gerbes de fleurs ", « des chaises ", « des pupitres », « quelques dessins sur un plan agrandi », « une grosse vis en laiton », «la nouvelle médiathèque »,...) et d'acteurs (« un public », « des anciens combattants », ...) qui renvoient à différents événements associés au monument aux morts (les commémorations, le déplacement de la statue, la transformation de l'espace environnant, sa protection en tant qu'œuvre d'un sculpteur). En dessous, un texte décrit les liens identifiés au cours de l'enquête. Débute ensuite le récit des chantiers intervenus sur cet espace public et les modifications que cela entraîne pour le monument aux morts. Par exemple, pour permettre la tenue des commémorations malgré les perturbations provoquées par les chantiers, un protocole d'accord est signé entre l'ensemble des acteurs concernés par le monument, dont la diversité est mise au jour par 
ce protocole lui-même. Le CartoAttaches met en lumière comment, derrière la simplicité apparente de l'objet (une sculpture décorée de gerbes de fleurs) et son usage en tant que décor de célébration, de multiples pratiques et liens s'organisent autour de lui.

9 Le CartoAttaches ne ressemble en rien à une représentation cartographique au sens géographique. Les commanditaires parlent d'une "pelote de laine» ou d'une «toile d'araignée » pour le décrire. Il permet pourtant de situer l'objet (la statue du monument aux morts) dans son environnement physique et social. Il montre les différents maillons d'une chaîne de médiation qu'un événement modifie et révèle. Dit autrement, il fait apparaitre les réseaux de pratiques existants autour de l'objet, autant de pratiques et de réseaux sur lesquels s'appuyer pour monter de nouveaux projets, pour intervenir sur cet espace. En cartographiant ce qui relie des non-humains (la statue du monument aux morts) aux humains, et en mettant en évidence ce qui les fait agir, le CartoAttaches renvoie aux pratiques de cartographie autochtone selon lesquelles toute société a ses propres principes de représentation de son monde, de son univers, et de sa cosmogonie. Dans des sociétés de traditions orales (comme les sociétés autochtones du Canada), ces cartographies ne se traduisent pas nécessairement par des représentations visuelles (des cartes) mais peuvent être transmises par des pratiques performatives (la danse). Elles impliquent des conceptions du monde où des non-humains (les esprits) interviennent dans la gestion et le devenir des territoires (Hirt, 2009).

10 Le procédé semble bien compris par les commanditaires qui en décrivent les finalités, avec leurs propres mots, mais très proches de l'intention originelle. Ils redécouvrent cet objet urbain a priori banal, à travers le récit qui personnifie l'objet, avec son nom, son histoire, ses relations, son avenir. Cette statue n'est plus seulement un objet rappelant la guerre mais apparaît comme un « lieu fédérateur, rassembleur, animé » (agent de la ville). Pour autant, les commanditaires disent leur scepticisme et leurs doutes quant à leur capacité à se saisir du CartoAttaches dans leur activité opérationnelle. Tout au long de l'intervention d'ArtPol, ils ont été déstabilisés dans leurs convictions (sur le public, le bien commun, le service public, la ville,...) et prennent conscience de certains préjugés sous-tendant leurs interventions. Le CartoAttaches représente une complexité qu'ils ne soupçonnaient pas, là où les modes de représentation habituels (notamment cartographiques) tendent à réduire la complexité pour la rendre appréhendable. Ce faisant, le CartoAttaches conduit les professionnels à interroger leurs propres perceptions et systèmes de représentations des espaces qu'ils administrent.

\section{La carte comme instrument d'éducation populaire}

11 En 2009, le CAUE (Conseil Architecture, Urbanisme, Environnement) d'un département d'î le de France lance un concours d'idées pour soutenir des interventions temporaires dans les espaces publics d'opérations de renouvellement urbain. Un des lauréats est le collectif AlterArchi, qui réunit de jeunes architectes, paysagistes et urbanistes qui mobilisent des démarches artistiques pour questionner et critiquer les pratiques habituelles des professionnels de la ville, à l'instar de nombreux collectifs apparus ces dernières années (Macaire, 2009). Ils proposent des démarches alternatives d'intervention auprès des habitants, qui se nourrissent de diverses influences, dont leurs propres pratiques artistiques. Par exemple, au début de leur intervention, un comédien interprète le personnage d'un jardinier-voyageur qui suscite des rencontres avec les habitants pour explorer et découvrir le quartier. Leur projet, la Jardinière, a pour objet la réalisation, 
avec les habitants, d'un jardin partagé conçu comme un lieu de rencontre dans un objectif d'éducation populaire. La conception, la construction et la gestion du jardin servent de prétexte à un projet de développement social qui vise à augmenter les capacités des habitants pour comprendre, proposer, et agir sur les mutations en cours dans leur quartier. Parler du quartier, discuter de pratiques quotidiennes et de préférences personnelles, découvrir et faire découvrir des lieux, construire une perception nuancée des atouts et problèmes du quartier, développer des nouvelles compétences pour agir et améliorer son quotidien sont les composantes des différentes activités conçues et mises en place par AlterArchi. La carte en est un des moyens principaux, mobilisée de différentes manières.

Il s'agit d'abord d'apprendre à se servir de la carte comme un outil d'orientation pour se situer dans la ville et à se repérer dans l'espace environnant. Lors de l'atelier Panorama, qui se déroule dans un appartement situé au dernier étage d'une tour vouée à la démolition, les habitants sont invités à dessiner le paysage sur les baies vitrées et de confronter ce panorama à une carte pour identifier les lieux par un exercice d'azimuts. Le jeu entre la vue réelle, le dessin et la carte est au cœur des échanges entre les participants, sous une forme ludique supposée faciliter la participation des habitants. La démarche, fondée sur le savoir-faire professionnel d'AlterArchi, vise à comprendre la relation que tissent les habitants avec leur territoire, tout en les formant aux techniques de représentation des professionnels.

Après cette première étape d'apprentissage, la carte sert d'instrument pour la production collective de connaissances sur le quartier. Autour d'elle, une conversation s'engage. Evocation de souvenirs relatifs à un site qu'elle représente. Localisation sur la carte d'un lieu auquel on pense. Le raccourci pour aller à la gare, la meilleure boulangerie, l'endroit secret d'un premier baiser... La carte se transforme, enrichie des connaissances subjectives produites ensemble. Elle est modifiée au fur et mesure, selon les remarques, critiques ou suggestions des différents participants. Elle devient le support d'un imaginaire partagé par lequel les habitants redécouvrent leur cadre de vie et modifient la représentation qu'ils en ont.

La carte devient enfin un outil de représentation de cet imaginaire, à travers la production d'un guide touristique diffusé ensuite auprès des habitants et des professionnels concernés par l'opération de renouvellement urbain. Conçu et fabriqué avec les habitants, le guide rend compte du travail mené pendant les activités mises en place par AlterArchi. Le guide ne cherche pas à être exhaustif et assume son hétérogénéité et sa subjectivité, tant dans la forme (photos, textes, mots, dessins) que sur le fond (lieux, vécu, impressions, ...). La multiplication des formes visuelles utilisées (façades, perspectives, plans, dessins d'enfants...) tranche avec les représentations cartographiques institutionnelles. Sans s'en tenir à de simples descriptions, les illustrations racontent des histoires (de lieu ou de personnes), révélant la diversité des perceptions et usages du quartier. Pour autant, la carte produite dans le guide reprend certaines conventions propres à la représentation géographique: une légende, une orientation, certains codes sémiologiques (le bleu de l'eau, le vert de la nature), un jargon technique (avec des catégories comme " espace public », « centralité »).

15 La Jardinière contribue à la co-construction, à travers la constitution d'un petit groupe d'habitants, d'une nouvelle relation à sa ville et de nouvelles compétences (faire un herbier, construire du mobilier urbain, lire une carte, se repérer dans le paysage, faire un guide, etc.). La carte est utilisée comme un outil pour saisir les perceptions des habitants 
pour agir sur les représentations de ce territoire, en mettant en valeur les connaissances informelles et sensibles des habitants rejoignant ainsi un des principes de la cartographie participative (Palsky, 2013). Le terme cartographie participative recoupe des pratiques cartographiques variées, mobilisant les habitants d'un territoire, grâce à l'intermédiation d'un opérateur spécialisé militant et/ou professionnel (ici, AlterArchi). Elles sont des productions collectives de représentation cartographique d'un territoire, s'appuyant sur les savoirs, pratiques et usages des habitants. L'enjeu n'est alors pas de fournir des informations homogènes et objectivables sur l'ensemble du territoire mais de rendre compte de la manière dont il est vécu et perçu par ses usagers à travers des histoires intimes des habitants. Dans les dispositifs de concertation, la cartographie participative vise à impliquer les habitants dans la conception de la représentation d'un problème ou d'un projet. La carte agit alors comme un médiateur qui met en évidence les points de vue habitants face aux institutions (Hirt, Roche, 2013). Si la cartographie participative est porteuse d'idéaux d'émancipation, plusieurs auteurs soulignent les paradoxes de ces dispositifs perçus comme des instruments de l'assimilation culturelle (notamment dans des contextes post-coloniaux) qui imposent une sémiologie officielle à des formes autochtones de cartographie (Hirt, Roche, 2013). Ces dispositifs sont portés par des professionnels dont les compétences spatiales et cartographiques initiales entretiennent un déséquilibre, voire une hiérarchie des positions face aux usagers participants (Nonjon, Liagre, 2012; Palsky, 2013). Si ces cartographies sont un outil de mobilisation collective et un moyen de faire valoir le point de vue des habitants, elles sont aussi un instrument de reconnaissance des professionnels de la participation car elles sont devenues une prestation parmi d'autres du répertoire d'action de la concertation (Nonjon, Liagre, 2012).

La carte du guide touristique produite dans le cadre de la Jardinière est révélatrice de ces ambiguïtés. La production finale de la représentation visuelle de la carte est le fait des professionnels d'AlterArchi qui maîtrisent les conventions graphiques (même s'ils les détournent) et une capacité d'analyse et de représentation des informations produites par les habitants. Valorisant des savoirs subjectifs (« là où on aime prendre le soleil », « là où tout le monde passe »), mobilisant des outils de représentation variés (récits, dessin d'enfants), elle reprend aussi des catégories d'analyses des professionnels (le zonage, les modes et axes de transport, les équipements publics), les conventions de la carte géographique (légende, orientation,...) et certains termes techniques ( la centralité commerciale ", « les espaces publics ",...). La présence d'artistes dans le collectif et leur propre sensibilité artistique n'effacent pas leurs automatismes professionnels incorporés. La carte traduit cette hybridation entre perceptions sensibles et technicité de la représentation. D'autre part, les protocoles d'intervention et méthodologies de travail avec les habitants qu'inventent AlterArchi pour la Jardinière, ont ensuite été repris et reformulés dans d'autres cadres. En un sens, la Jardinière est pour eux le projet de leur propre émancipation des codes professionnels habituels, par lequel ils deviennent des professionnels de l'urbanisme proposant d'autres modalités d'intervention.

\section{La cartographie numérique à l'épreuve de la gestion de données}

17 CitéNum, projet artistique développé depuis 2007 par ArtNum, association culturelle spécialisée dans l'accompagnement de projets numériques, a pour terrain d'expérimentation un campus francilien. Son objectif est de développer des projets 
artistiques qui utilisent des technologies numériques pour interroger les mutations de la ville et la place du citoyen. Une base de données territoriales coordonnée, gérée et exploitée par ArtNum alimente les projets artistiques développés sur ce territoire et est alimentée par les artistes qui proposent des collectes de données spécifiques. Un partenariat est noué avec le gestionnaire du campus pour développer des outils de valorisation du patrimoine, comme par exemple NumériCarte.

NumériCarte est une carte numérique qui utilise une photographie aérienne et un outil de cartographie d'un célèbre opérateur de l'internet comme base pour géolocaliser les données numériques et les rendre accessibles en ligne. Les usagers de l'interface peuvent questionner les contenus mis en ligne par ArtNum, les filtrer avec des mots-clés ou chercher des nouveaux contenus sur des réseaux sociaux. NumériCarte se distingue des autres plateformes numériques par la nature de ses contenus. Elle regroupe des documents utilisés habituellement par les professionnels de l'urbanisme (documents d'archives, photos, vidéos, enregistrements sonores, études d'urbanisme, cartes,...) ainsi que des données et des œuvres produites par des artistes et ArtNum. La valorisation de ces données nécessite un travail de sélection, de numérisation, d'indexation et de transformation pris en charge par ArtNum. Sont privilégiées les données qu'ArtNum qualifie d'«insolites", de "subjectives» ou de "sensibles», par volonté de se distinguer des données géographiques ou institutionnelles.

«On ne va pas chercher l'objectivité du plan large, mais on va chercher le petit détail, la petite micro histoire. » (membre d'ArtNum)

19 Les choix de données opérés par ArtNum sont contraints par des considérations juridiques (droit d'auteur et droit à l'image), techniques (fonctionnement des interfaces) et esthétiques (exigences de certains artistes), freinant l'alimentation de la base par d'autres usagers. La deuxième caractéristique de NumériCarte est la nature des métadonnées utilisées pour l'indexation (ou mot-clés). La gestion de la base de données nécessite en effet un travail laborieux d'indexation des données, qui consiste à associer les données à des mots-clés qui les décrivent. Ce travail est incontournable pour qu'une donnée puisse être utilisée par les interfaces et accessible aux usagers. Mais, comme dans tout processus de catégorisation, se pose la question des choix des mots-clés. Une large part est laissée aux appréciations subjectives d'ArtNum et renvoie à ses propres visions du territoire et à la finalité de NumériCarte (la valorisation du patrimoine).

Le choix d'une cartographie numérique comme mode de représentation territoriale n'est pas anodin. Il est assumé et défendu par ArtNum pour qui ce serait le seul moyen de développer des projets artistiques territorialisés et de pouvoir accéder à une multiplicité de données territorialisées. La carte est aussi considérée comme un outil facilement appropriable par les acteurs du territoire. ArtNum insiste ainsi auprès des artistes mobilisés pour favoriser NumériCarte comme mode de restitution de leur travail. L'appropriation de cette consigne par les artistes est variable. Dans la plupart des projets artistiques, cet usage de la carte se traduit par la géolocalisation de l'œuvre ou d'un élément de l'œuvre. NumériCarte se présente in fine comme un ensemble de points de différentes couleurs sur une carte, rejoignant les critiques de la webcartographie. L'essor des technologies de l'information géographique avait en effet suscité l'espoir d'une démocratisation de la production des savoirs géographiques et l'émergence de nouvelles cartographies numériques participatives, alimentées par des utilisateurs concernés. Or les opérateurs dominants du géoweb encouragent une logique de contribution (volontaire ou non) qui se limite à visualiser la localisation d'une information (comme NumériCarte) 
sans inventer de nouveaux processus de production et de représentation de l'information géographique (Mericskay, Roche, 2011; Joliveau, Noucher et al., 2013).

D'autre part, ces cartographies numériques requièrent, contrairement à certaines idées reçues, des compétences techniques qui limitent l'étendue de la participation, réservées à quelques géo-geeks (Hirt, Roche, 2013). Comme d'autres formes d'art numérique (Fourmentraux, 2008), NumériCarte mobilise des compétences nouvelles, celles des informaticiens, qui participent à la conception de l'interface. Ces nouvelles collaborations font apparaitre de nouveaux espaces de négociation entre les artistes et les informaticiens, auxquels s'ajoutent ici les enjeux portés par ArtNum et par le gestionnaire du campus qui encadrent le choix des données numérisées ainsi que leur indexation. De part la diversité des intervenants, des enjeux, des intérêts et des contraintes, les artistes apparaissent finalement comme des producteurs secondaires, de simples contributeurs de NumériCarte. D'ailleurs certains s'en détournent, comme des artistes sonores qui ont accepté de travailler sur une cartographie sonore, mais ont délaissé NumériCarte pour lui préférer une plateforme spécifique, utilisée par la communauté des artistes sonores qui enrichissent collectivement le travail réalisé, à partir d'un protocole d'utilisation précis. Plus qu'une cartographie participative (où les usagers contribuent aux réflexions sur les enjeux soulevés et leur représentation, ou l'émergence d'un contreprojet), NumériCarte relève de la cartographie contributive, restreinte ici aux artistes sollicités par ArtNum. Conscient de ces limites, ArtNum est en recherche de nouveaux projets éditoriaux, mais la lourdeur du processus de numérisation et d'indexation contraint son interactivité et son déploiement pour d'autres usages.

\section{Conclusion}

L'analyse de ces différentes interventions artistiques aboutit à des conclusions mitigées. Les artistes utilisent la cartographie pour rendre compte de complexités de différentes natures, qu'il s'agisse de la difficulté à décider collectivement d'une limite, des pluralités de pratiques d'un objet apparemment simple, des différents usages et perceptions d'un quartier, de la mise en cohérence d'une multitude de données. L'usage qu'ils en font diffère des pratiques habituelles des acteurs du territoire, tant dans la forme que dans le contenu. Ces différences résultent, en partie, de la volonté des artistes (ou de la demande explicite qui leur est faite) d'adopter une posture en décalage avec les habitudes et pratiques habituelles des professionnels de l'urbanisme. On peut faire l'hypothèse que ces différences s'expliquent aussi par le fait que ces artistes travaillent en suivant leur propre perception d'une réalité, sans chercher à se justifier, à raisonner ou à objectiver leurs ressentis. Ils stimulent également cette forme d'expression auprès de leurs interlocuteurs - professionnels de l'urbanisme ou habitants. De ce fait, les cartographies qu'ils proposent relèvent moins d'une représentation de la réalité physique de l'espace que d'une représentation de sa réalité subjective. Cette posture revient à considérer que celle-ci participe tout autant de la réalité de l'espace que sa seule matérialité. Ce faisant, l'attention portée par les artistes aux réalités subjectives de l'espace (re)questionne la formulation des problèmes et enjeux de l'espace considéré. Ces détournements volontaires interrogent ainsi l'usage et la production des cartes comme outil conventionnel de l'action territoriale. Ils convergent avec les critiques à l'encontre des pratiques cartographiques selon lesquelles la carte est une production sociale qui résulte de choix opérés par les acteurs, selon des systèmes de ressources et de contraintes, mais 
également selon des intérêts et des enjeux. En cela, les propositions cartographiques des artistes rappellent que la carte doit être manipulée avec conscience.

\section{BIBLIOGRAPHIE}

AMILHAT SZARY A.-L. (2012), «The Geopolitical Meaning of a Contemporary Visual Arts Upsurge on the Canada-US Border ", International Journal, Canada's Journal of Global Policy Analysis, 67, 4, pp. 951-962.

ARDENNE P. (2002), Un art contextuel - Création artistique en milieu urbain, en situation, d'intervention, de participation, Paris, Flammarion.

BLANC N. (2008), Vers une esthétique environnementale, Paris, Quae.

CHAUDOIR P. (2000), Discours et figures de l'espace public à travers les « arts de la rue », La ville en scènes, Paris, L'Harmattan.

DEBARDIEUX B., LARDON S. (dir.) (2004), Les figures du projet territorial, La Tour d'Aigues, éditions de l'Aube.

FLICHY P. (2010), Le sacre de l'amateur. Sociologie des passions ordinaires à l'ère numérique, Paris, La république des idées, Seuil.

FOURMENTRAUX J.-P. (2008), « đuvrer en commun. Dilemmes de la création interdisciplinaire négociée », Négociations, 10, pp. 25-39.

GONON A. (2011), «La portée disruptive des Arts de la ville: l'exemple du groupe "Ici Même" (Paris) », in ANSON P. (dir.), Les Arts de la ville dans le projet urbain. Débat public et médiation, Tours, Presses universitaires François Rabelais, pp. 313-323.

GRÉSILLON B. (2008), « Ville et création artistique. Pour une autre approche de la géographie culturelle », Annales de Géographie, 660-661, pp. 179-198.

HIRST I., ROCHE S. (2013), « Cartographie participative », in CASILLO I., BARBIER R., BLONDIAUX L. et al. (dir.), Dictionnaire critique et interdisciplinaire de la participation, Paris, GIS Démocratie et Participation, [en ligne] http://www.participation-et-democratie.fr.

HIRT I. (2009), « Cartographies autochtones. Eléments pour une analyse critique », L'espace géographique, 38, 2, pp. 171-186.

JOLIVEAU T., NOUCHER M., ROCHE S. (2013), « La cartographie 2.0, vers une approche critique d'un nouveau régime cartographique », L'Information géographique, 77, 4, pp. 29-46.

LASCOUMES P. (2007), « Gouverner par les cartes », Genèses, 68, pp. 2-3.

LATOUR B. (2006), Changer de société, refaire de la sociologie, Paris, La Découverte.

LE BOURHIS J.-P. (2007), « Du savoir cartographique au pouvoir bureaucratique. Les cartes des zones inondables dans la politique des risques (1970-2000) », Genèses, 68, pp. 75-96.

MACAIRE E. (2009), « Des architectes dans le champ socio-culturel », in BUREAU M.-C., PERRENOUD M., SHAPIRO R. (dir.), L'artiste pluriel. Démultiplier l'activité pour vivre de son art, Villeneuve d'Ascq, Presses Universitaires du Septentrion, pp. 161-174. 
MEKDJIAN S., AMILHAT SZARY A.-L., MOREAU M. et al. (2014), « Figurer les entre-deux migratoires. Pratiques cartographiques expérimentales entre chercheurs, artistes et voyageurs ", Carnets de géographes 7, [en ligne], http://www.carnetsdegeographes.org/carnets_terrain/ terrain_07_01_Mekdjian.php.

MERICSKAY B., ROCHE S. (2011), « Cartographie 2.0 : le grand public, producteur de contenus et de savoirs géographiques avec le web $2.0 »$, Cybergeo, [en ligne] http://cybergeo.revues.org/24710

NONJON M., LIAGRE R. (2012), « Une cartographie participative est-elle possible? Ressorts et usages de la "cartographie" dans les projets d'aménagements urbains », EspacesTemps, [en ligne] : http://www.espacestemps.net/articles/une-cartographie-participative-est-elle-possible/.

O'ROURKE K. (2013), Walking and Mapping. Artists as Cartographers, Cambridge, Ma, MIT Press.

PALSKY G. (2013), « Cartographie participative, cartographie indisciplinée », L'Information géographique, 77, 4, pp. 10-25.

VOLVEY A. (dir.) (2007), « Spatialités de l'art », (numéro thématique), Travaux de l'Institut de Géographie de Reims, 33, pp. 129-130.

\section{NOTES}

1. Cet article rend compte des résultats d'une recherche-action en cours soutenue par le Conseil Régional Ile-de-France. Il se base sur l'analyse approfondie de quatre cas : entretiens avec les différentes parties prenantes, observations participantes (pour deux cas), analyse des documents produits. Dans les exemples choisis, les artistes interviennent aux côtés des professionnels de l'urbanisme sur un problème que ceux-ci se posent. La recherche porte sur les modalités de cette collaboration nouvelle. Tous les éléments ont été anonymisés. Pour respecter cet anonymat, l'article ne présente pas d'illustrations du travail de ces artistes.

2. Les auteurs remercient les évaluateurs d'avoir attiré leur attention sur ce point.

\section{RÉSUMÉS}

Cet article explore de nouvelles approches de l'intervention artistique en lien avec le territoire, où l'intervention de l'artiste ne vise plus à faire œuvre mais à contribuer aux réflexions sur les transformations territoriales en cours ou à venir. Dans les quatre cas étudiés, l'usage de la cartographie apparaît comme une des modalités de l'intervention des artistes : mise à l'épreuve sur le terrain des cartes de planification; analyse d'un espace par une cartographie des liens le reliant à son environnement; formation des habitants à la lecture et à la production de carte ; mise en place d'une carte numérique interactive. En présentant chaque exemple dans son contexte d'intervention, il s'agit d'interroger l'usage que font les artistes de la carte. Comment les artistes utilisent-ils la cartographie? Les interventions des artistes seront étudiées ici au regard des controverses sur la cartographie comme construction sociale porteuse de conventions plus ou moins négociées. La carte étant un instrument conventionnel de représentation et de 
collaboration des professionnels de l'action territoriale, leurs réactions révèlent si et en quoi les propositions cartographiques de ces artistes renouvellent les controverses sur la cartographie.

This paper explores new types of artistic interventions developed in relation with urban space or city planning activities. Here, artists do not only aim to create artwork but also to contribute to work on on-going or future territorial transformations, along with urban professionals. In the four cases studied, cartography appears as one of the means used by artists to work: testing the validity of planning documents in situ; analysing an urban space by mapping its ties with its environment; training inhabitants to the reading and the making of maps; implementing a digital map. We will analyse each example separately to question the way artists make use of maps. These artistic interventions will be studied in view of the controversies on cartography. Cartography is considered here as a social construction based on conventions more or less negotiated. Maps are conventional tools of representation and collaboration for urban professionals, their reactions show if and how the use made of maps by artists renew controversies on cartography.

\section{INDEX}

Mots-clés : artiste, carte, projet urbain, cartographie participative

Keywords : artist, map, urban project, participative cartography

\section{AUTEURS}

\section{ELSA VIVANT}

Université Paris-Est, LATTS, UPEM, CNRS, Ecole des Ponts, F-77454, Marne la Vallée, Elsa.vivant@univ-paris-est.fr

\section{BURCU OZDIRLIK}

Université Paris-Est, Lab’Urba, UPEM, UPEC, F-77454, Marne la Vallée, burcuozdirlik@yahoo.com

\section{NADIA ARAB}

Université Paris-Est, Lab'Urba, UPEM, UPEC, F-77454, Marne la Vallée, nadia.arab@univ-parisest.fr 